\title{
Utility of fluorescence in situ hybridization in sub- classifying unclassified high-grade sarcomas: A study of 40 cases using break-apart probes of EWSR1, FOXO1A, SS18 and DDIT3 genes
}

\author{
Alfredo L. Valente, J amie Tull, Shengle Zhang \\ Department of Pathology, SUNY Upstate Medical University, Syracuse, New York 13210, U.S.A \\ Correspondence: Shengle Zhang. Department of Pathology, SUNY Upstate Medical University, Syracuse, New York \\ 13210, U.S.A. Telephone: 1-315-464-7131. E-mail: zhangs@upstate.edu
}

Received: November 16, 2011 Accepted: January 10, $2012 \quad$ Published: April 1, 2012

DOI : $10.5430 /$ jst.v2n2p4 URL: http://dx.doi.org/10.5430/jst.v2n2p4

\section{Abstract}

Objectives: In an era of rapid advances in oncologic treatment, there is continuous emphasis for a definitive classification of undifferentiated sarcomas in order to select the most appropriate therapeutic regimens against these malignancies.

EWSR1, FOXO1A, SS18 and DDIT3 gene break-aparts associated with chromosomal translocations are widely used as specific molecular markers in diagnosing the Ewing family of tumors, alveolar rhabdomyosarcoma, synovial sarcoma and myxoid liposarcoma, respectively. However, the utility of these markers for definitive categorization of previously unclassified high-grade sarcomas has not been studied.

Methods: We identified 40 sarcomas from 2003 to 2009, which on light microscopy exhibited a spindle cell or epithelioid morphology with marked nuclear atypia, high mitotic rate and necrosis. These sarcomas were high grade and poorly differentiated, and remained unclassified despite the use of immunohistochemical panels comprising neural, smooth muscle, skeletal muscle, melanocytic, epithelial, vascular and fibrohistiocytic markers.

Results: Thirty five cases were resection specimens, and the rest were needle biopsies. Fluorescence in situ hybridization (FISH) was applied to formalin fixed paraffin embedded tissue (FFEP) with break-apart probes for EWSR1, FOXO1A, SS18 and DDIT3 gene. In positive cases, RT-PCR was done to detect specific gene fusions associated with translocations. One of 40 cases was positive for DDIT3 gene break-apart, consistent with a dedifferentiated myxoid liposarcoma.

Conclusions: Subsequent reverse transcriptase (RT)-PCR demonstrated a FUS-DDIT3 fusion, supporting the FISH result. Another case was positive for the FOXO1A gene break-apart, consistent with alveolar rhabdomyosarcoma, even though RT-PCR failed to reveal specific gene fusion. Although with low frequency, FISH using a variety of probes can provide precise classification in a few otherwise unclassifiable high grade sarcomas. The findings also affirm the utility of FISH technology in FFPE tissues, including small biopsies.

\section{Key words}

EWSR1, FOXO1A, SS18, DDIT3, Fluorescence in situ hybridization, High-grade sarcoma 


\section{I ntroduction}

Classifying soft tissue sarcomas is important for patient management since the assorted sarcomas are associated with different clinical prognosis and therapeutic responses. Ewing sarcoma and rhabdomyosarcoma showed good response to vincristine/ actinomycin D/cyclophosphamide plus ifosfamide/etoposide ${ }^{[1-3]}$. Synovial sarcoma responded better to doxorubicin plus ifosfamide ${ }^{[4]}$, while myxoid liposarcoma responded preferentially to trabectedine ${ }^{[1]}$. However, approximately $10 \%$ of sarcomas are poorly differentiated, and cannot be further classified based on conventional histology and immunohistochemistry ${ }^{[5]}$, making it difficult to select appropriate therapeutic regimens. With current developments in molecular technology, characteristic genetic alterations have been identified in a variety of soft tissue tumors ${ }^{[6]}$, which have become new biomarkers to facilitate tumor diagnosis and classification. Classification based on genetic abnormalities present in sarcomas can also be of potential benefit to targeted therapies ${ }^{[6-8]}$. EWSR1 gene rearrangements have been identified in a group of soft tissue tumors ("Ewing family of tumors"), which includes Ewing/PNET, clear cell sarcoma, desmoplastic small round cell tumor, extraskeletal myxoid chondrosarcoma and angiomatoid fibrous histiocytoma ${ }^{[6,9]}$. Therefore, fluorescence in situ hybridization (FISH) with EWSR1 break-apart probe can detect all the tumors with EWSR1 associated rearrangements. See Table 1. FOXO1A (FKHR), SS18 (SYT) and DDIT3 (CHOP) gene rearrangements are associated with alveolar rhabdomyosarcoma, synovial sarcoma and myxoid liposarcoma, respectively ${ }^{[6,9]}$, and therefore can be detected with corresponding break-apart FISH probes. Although FISH has been used for diagnosis in a variety of soft tissue sarcomas ${ }^{[10]}$, the utility with these probes in sub-classifying the unclassified high-grade sarcomas has not been evaluated. In our study, 40 cases of formalin-fixed, paraffin-embedded (FFPE) high-grade sarcomas were tested with FISH probes for EWSR1, FOXO1A, SS18 and DDIT3 gene break-aparts. RT-PCR was performed to identify specific gene fusions in the cases with presence of gene break-apart.

\section{Methods}

\section{Case Materials}

From the Pathology archives of SUNY Upstate Medical University, we identified 40 sarcoma cases, diagnosed from 2003 to 2009, presenting with a high-grade spindle cell or epithelioid morphology and no definitive evidence of differentiation (Table 2). Tumors had been graded according to the French National Federation of Cancer Centers (FNCLCC) grading system. Twenty two patients were male, and 18 were females (male/female ratio: 1.2:1.0). Patient's age ranged from 15 to 96 years, with a median age of 64 years. The lower extremity was the most common location with 26 cases (14 of them were located in the thigh), followed by the upper extremity (9 cases), trunk (4 cases), and lung (1case). Hematoxylin and eosin-stained sections were reviewed by two pathologists (ALV, SZ), with inter-observer agreement in all cases. The selected sarcomas remained unclassified after immunohistochemistry using neural, smooth muscle, skeletal muscle, melanocytic, epithelial, vascular, smooth muscle and fibrohistiocytic markers. The majority of these cases (87.5\%) were resection specimens, and the remainder $12.5 \%$, core needle biopsies. Two tissue microarrays were constructed with the resection specimens to improve efficiency and save reagents.

\section{FISH}

FISH with break-apart probes for EWSR1, FOXO1A, SS18 and DDIT3 were performed in paraffin-embedded tissue, which is the most available specimen in pathology. The tissues were cut in 3-5 micron sections on a microtome. The sections were placed on positive charged slides, deparaffinized in Xylene (3 times, 5 minutes each), placed in ethanol (2 times, 1 minute each), and then dried. They were then pretreated with boiled citrate buffer ( $\mathrm{pH}$ 6.0) for 10 minutes followed by protease digestion for another 20 minutes. Probes (Abbott Molecular Inc, Des Plaines, IL) were then applied before putting on a coverslip. The slides were placed on a HYBrite, denatured at $73^{\circ} \mathrm{C}$ for 5 minutes, and then hybridized at $37^{\circ} \mathrm{C}$ overnight. Following hybridization, the membranes were solubilized by washing and then counterstained with DAPI I, a nucleic-specific stain. The slides were examined using a fluorescence microscope with appropriate excitation 
and emission filters that allow visualization of Spectrum Orange, Spectrum Green, and DAPI fluorescence. Hybridization signals were counted in at least 40 morphologically intact, non-overlapping nuclei. In normal cells with lack of break-apart rearrangement, a two-fusion signal (yellow) pattern was observed, which reflects two intact copies of gene. Cells with break-apart showed one fusion (red and green overlapping), one green, and one orange signals. A break-apart signal to intact signal ratio was obtained. Based on our previous assay validation, a ratio of break-apart to intact signals greater than 0.10 was considered positive for FOXO1A, SYT and DDIT3 probes, and 0.2 for EWSR1 probes.

\section{RT-PCR}

RNA was extracted from paraffin sections using Optimum FFPE RNA Isolation kit (Zymo Research, Orange, CA, USA). Primers for variants of FUS-DDIT3 fusions were listed in Table 3. Consensus primers for both PAX3-FOXO1 and PAX7-FOXO1 were 5'-CMGACASCAGCTCTGCCTAC-3' (PAX-7M, forward) and 5'-GGACAGATTATGACGAATTGAA-3' (FKHR-2M, reverse), with amplicon of 143 bp and 155 bp, respectively. Primers for glucose-6-phosphate dehydrogenase (G6PD) were used as an internal control. RT-PCR was performed using Qiagen OneStep RT-PCR kit (Qiagen, Valencia, CA), with a $50 \mu \mathrm{L}$ of reaction mixture containing $0.6 \mu \mathrm{M}$ of all primers on Gene Amp® PCR System 9700. The instrument was set up as follows: $30 \mathrm{~min}$ at $50^{\circ} \mathrm{C}$ for reverse transcription, $15 \mathrm{~min}$ initial PCR activation step at $95^{\circ} \mathrm{C}$, followed by 40 cycles of 30 s of denaturation at $94^{\circ} \mathrm{C}$, 30s of annealing at $60^{\circ} \mathrm{C}, 1 \mathrm{~min}$ of extension at $72^{\circ} \mathrm{C}$, and a final extension of $10 \mathrm{~min}$ at $72^{\circ} \mathrm{C}$. The PCR products were electrophoresed on $10 \%$ polyacrylamide $1 \mathrm{X}$ TBE gel, stained in ethidium bromide, and visualized under a UV lamp.

\section{Results}

FISH assays with 4 probes on all 40 cases (160 samples in total) showed satisfactory fluorescent signals. The assay with EWSR1 and SS18 break-apart probes did not reveal genetic abnormalities in any case. One case (case 11) tested positive with the DDIT3 break-apart probe (Figure 1a, b), and another one (case 14), with the FOXO1A break-apart probe (Figure 2a, b). Subsequent RT-PCR showed a characteristic FUS-DDIT3 of myxoid liposarcoma in case 11(Figure 3). In case 14, PAX3-FOXO1A and PAX7-FOXO1A fusions could not be revealed by our RT-PCR (Figure 3). However, the test sensitivity of RT-PCR used is only $80 \%$, i.e. approximately $20 \%$ of alveolar rhabdomyosarcomas would not be detected.

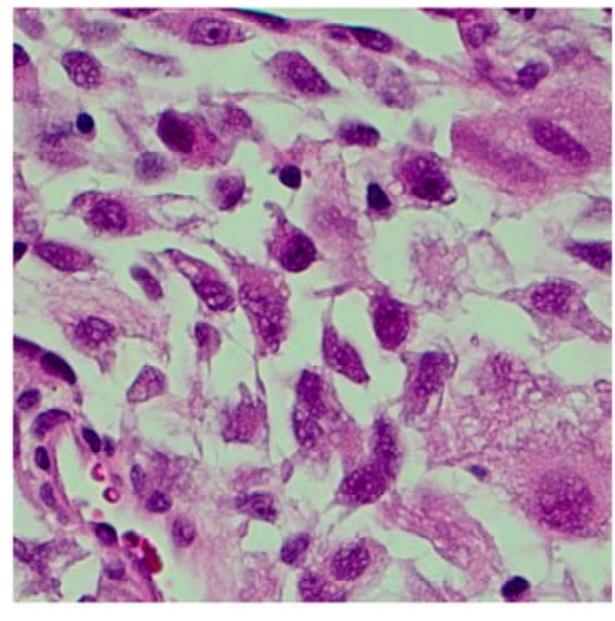

Figure 1a

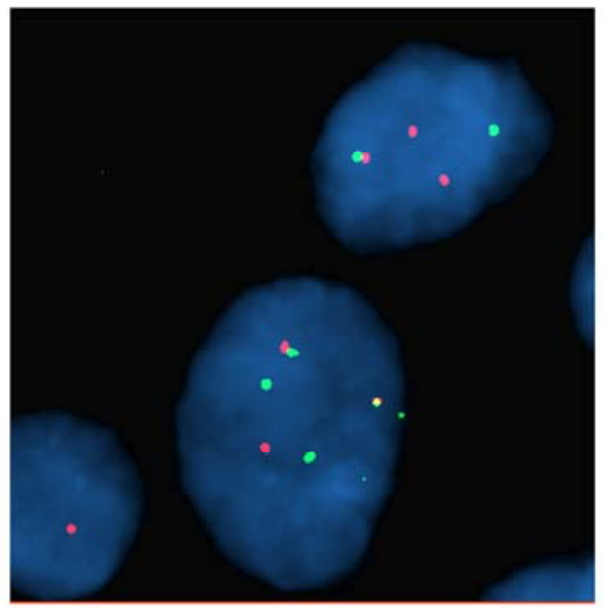

Figure 1b

Figure 1. (a). H\&E stain of case 11 shows high-grade round cell sarcoma in absence of lipoblasts and myxoid changes seen in conventional myxoid liposarcoma. (b). FISH shows DDIT3 gene break-apart, a characteristic genetic alteration in myxoid liposarcoma. Polysomy is present. 


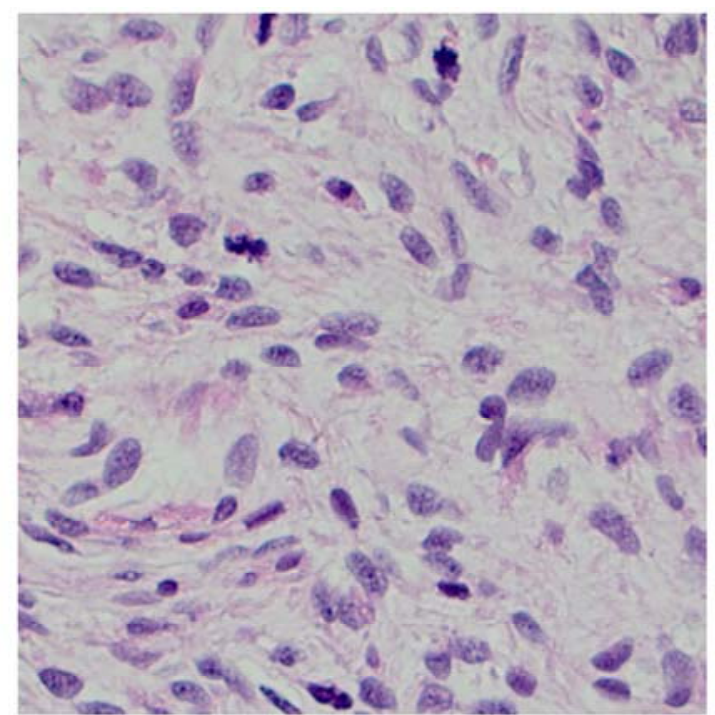

Figure 2a

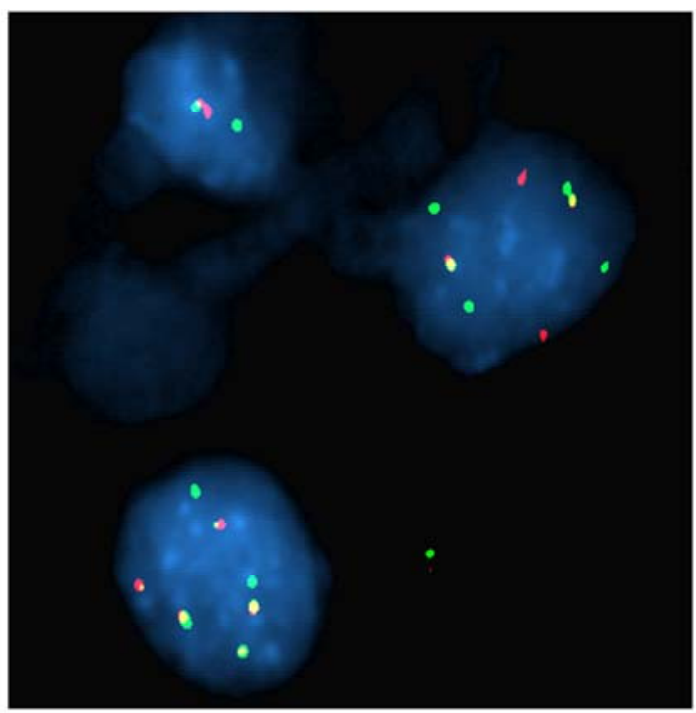

Figure $2 b$

Figure 2. (a). H\&E stain of case 14 shows high-grade spindle cell sarcoma in absence of features of "small blue round cells" seen in conventional alveolar rhabdomyosarcoma. (b). FISH shows FOXO1A gene break-apart, a characteristic genetic alteration in alveolar rhabdomyosarcoma. Polysomy is present.

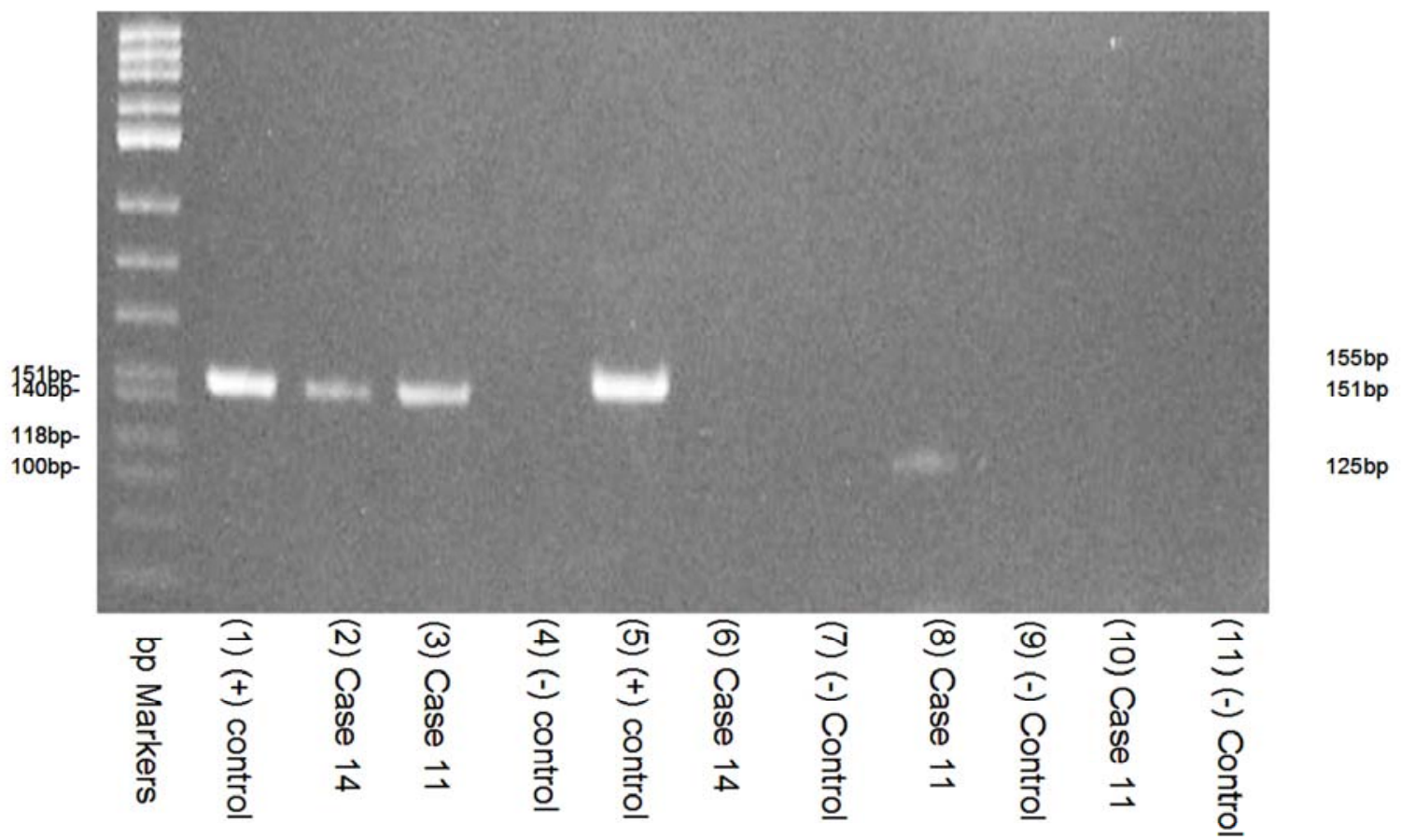

Figure 3. RT-PCR assay on case 11 (with DDIT3 break apart), and case 14 (with FOXO1A break-apart). Lanes 1-4: primers for G6PD gene as control; Lanes 5-7: primers for PAX3/7-FOXO1 fusions; Lanes 8-9: primers for FUS-DDIT3, types 1, 3; Lanes 10-11: primers for FUS-DDIT3, types 2, 4. Case 11 (lane 8) shows positive for type 1 FUS-DDIT3 gene fusion (125bp amplicon). Case 14 (lane 6) shows no PCR product with primers for PAX3/7-FOXO1. 


\section{Discussion}

Classification of sarcomas is essential for oncologists to select appropriate therapeutic regimens ${ }^{[1-4]}$. In recent years, the diagnosis of soft tissue tumors has become increasingly reliant on molecular analysis, predominantly due to overlapping morphologic features and immunohistochemical profiles in many of these neoplasms ${ }^{[9,11]}$. In addition, soft tissue sarcomas may be susceptible to molecular targeted therapies based on their genetic alterations, especially gene fusions associated with chromosomal translocations and mutations ${ }^{[7,8]}$.

Our study was performed on 40 cases of high-grade sarcomas that could not be further classified, either by morphology or immunohistochemistry. One case (case 11) showed DDIT3 break-apart, and based on this result, it was reclassified as a myxoid liposarcoma that underwent dedifferentiation. In addition, the FUS (TLS)-DDIT3 gene fusion was identified by RT-PCR, confirming the characteristic $\mathrm{t}^{[12,16]}$ (q13; p11) translocation present in myxoid liposarcomas. In this case, the initial biopsy revealed a poorly differentiated tumor with extensive necrosis, infiltrating the skeletal muscle. Malignant cells exhibited an epithelioid morphology, with significant nuclear pleomorphism including bizarre forms. Numerous cells also showed prominent nucleoli and abundant eosinophilic cytoplasm. Some cells contained cytoplasmic vacuoles, but typical features of lipoblasts, such as nuclear indentation or scalloping, were not identified (Figure 1). The stroma was predominantly myxoid. Immunohistochemistry was negative for skeletal and smooth muscle markers (Myo-D1, myogenin, and muscle specific actin), neural/melanocytic marker S-100 protein, and epithelial marker pancytokeratin. CD34, which usually stains endothelial cells and a variety of soft tissue neoplasms including solitary fibrous tumor/hemangiopericytoma and dermatofibrosarcoma protuberans, was also negative. This undifferentiated histology and lack of specific immunophenotype led to a previous diagnosis of high-grade sarcoma with myxoid features. Obviously, without current molecular tests, it would be impossible to re-classify it as dedifferentiated myxoid liposarcoma.

Another case (case 14) exhibited the FOXO1A break-apart, consistent with alveolar rhabdomyosarcoma. In this case, the initial core biopsy showed a hypocellular myxoid neoplasm with no specific immunophenotype. The resection specimen, however, disclosed a high-grade malignancy with hypercellular areas associated to a fibrous stroma, alternating with hypocellular areas in a myxoid background, and composed of atypical cells with markedly pleomorphic spindle and epithelioid type nuclei, including numerous multinucleated forms, partially arranged in interlacing fascicles. In the cellular areas, a strikingly high mitotic rate, including tripolar and tetrapolar mitoses, was present. Morphologic features of alveolar rhabdomyosarcoma, such as alveolar pattern, were not identified. Immunohistochemistry, on the other hand, did not show any specific immunophenotype. MyoD1 (marker for rhabdomyocytes), muscle specific action, desmin and pancytokeratin were all negative, only CD34 was focally positive. A diagnosis of high-grade sarcoma consistent with malignant fibrous histiocytoma was then issued. Since FOXO1A break-apart has not been identified in any other sarcomas other than alveolar rhabdomyosarcoma ${ }^{[9,12]}$, this case was re-classified as alveolar rhabdomyosarcoma. Although a translocation partner was not revealed by RT-PCR, this does not exclude alveolar rhabdomyosarcoma since the test sensitivity is only $80 \%$. An unknown variant of FOXO1A associated gene fusion, which is not detectable by our primers, may cause the failure of our RT-PCR approach. Two new PAX3-NCOA1 and PAX3-FOXO4 gene fusions have been discovered in alveolar rhabdomyosarcoma ${ }^{[9]}$, and unlikely involves the case since FOXO1A break-apart is identified.

Interestingly, EWSR1 gene break-apart was not seen in any of the high-grade sarcomas, even though it involves a "family" of 6 types of sarcomas, i.e. Ewing/PNET, clear cell sarcoma, desmoplastic small round cell tumor, extraskeletal myxoid chondrosarcoma and angiomatoid fibrous histiocytoma (Table 1). One of the reasons may be the age group of our study is relatively old (mean 64), while most tumors of Ewing' tumor family occur in children or young adults. It is not surprising that SS18 break apart was not identified in any of the tumors since poorly differentiated synovial sarcoma is rare.

Despite the low frequency of the genetic alterations, the molecular approach is valuable in providing precise classification in a few otherwise unclassifiable high-grade sarcomas. Compared with RT-PCR, FISH assay is morphology-based with higher specificity, and easier to perform on small biopsies with sound reproducibility ${ }^{[10,12]}$. In addition, RT-PCR assay has lower clinical test sensitivity due to variable gene fusions that may not be completely covered by primers. Although FISH 
with break-apart probes cannot directly detect gene fusions as RT-PCR, it is an excellent surrogate for the detection of translocation-associated rearrangements ${ }^{[10,12]}$.

A significant proportion of high-grade sarcomas is composed of undifferentiated pleomorphic sarcomas/malignant fibrous histiocytomas (UPS/MFH), poorly differentiated leiomyosarcomas and malignant peripheral nerve sheath tumors (MPNST) in which genetic abnormalities with clinical utility have not yet been discovered ${ }^{[9]}$. At present time, FISH with available probes seems not to offer much diagnostic advantage in unclassified sarcomas due to low frequency ${ }^{[11]}$. But with current rapid advances in molecular oncology, development of new FISH probes should expand the utility of molecular tests in further categorization of unclassified high-grade sarcomas, and as such, gradually become part of routine diagnostic practice, especially in an era in which diverse chemotherapeutic protocols and target-specific therapies are being designed for the treatment of these malignancies ${ }^{[7,8,13]}$.

\section{References}

[1] Krikelis D, Judson I. Role of chemotherapy in the management of soft tissue sarcoma. Expert Rev Anticancer Ther. 2010;10:249-60. PMID: 20132000. doi:10.1586/era.09.176.

[2] Grier HE, Krailo MD, Tarbell NJ et al. Addition of ifosfamide and etoposide to standard chemotherapy for Ewing's sarcoma and primitive neuroectodermal tumor of bone. N Engl J Med. 2003;348:694-701. PMID: 12594313.

http://dx.doi.org/10.1056/NEJMoa020890

[3] Ruymann FB, Grovas AC. Progress in the diagnosis and treatment of rhabdomyosarcoma and related soft tissue sarcomas. Cancer Invest. 2000;18:223-41. PMID: 10754991. http://dx.doi.org/10.3109/07357900009031827

[4] Sleijfer S, Ouali M, van Glabbeke M et al. Prognostic and predictive factors for outcome to first-line ifosfamide-containing chemotherapy for adult patients with advanced soft tissue sarcomas: An exploratory, retrospective analysis on large series from the European Organization for Research and Treatment of Cancer-Soft Tissue and Bone Sarcoma Group (EORTC-STBSG). Eur J Cancer. 2010;46:72-83. PMID: 19853437. doi:10.1016/j.ejca 2009.09.022

[5] Slater O, Shipley J. Clinical relevance of molecular genetics to paediatric sarcomas. J Clin Pathol. 2007;60:1187-94. PMID: 17468291. doi:10.1136/jcp.2006.040113. http://dx.doi.org/10.1136/jcp.2006.040113

[6] Jain S, Xu RL, Prieto VG, et al. Molecular classification of soft tissue sarcoma and its clinical applications. Int J Clin Exp Pathol. 2010;3:416-29. PMID: 20490332.

[7] Wardelmann E, Schidhaus H-U, Merkelbach-Bruse S, et al. Soft tissue sarcoma: From molecular diagnosis to selection of treatment. Pathological diagnosis of soft tissue sarcoma amid molecular biology and targeted therapies. Ann Oncol. 2010;21 Suppl 7:vii265-269. PMID: 20943625. doi:10.1093/annonc/mdq381.

[8] Judson I. Targeted therapies in soft tissue sarcomas. Ann Oncol. 2010;21 Suppl 7:vii277-280. PMID: 20943628. doi: 10.1093/annonc/mdq288.

[9] Bridge JA, Cushman-Vokoun AM. Molecular diagnosis of soft tissue tumors. Arch Pathol Lab Med. 2011;135:588-601. PMID: 21526957.

[10] Tanas MR, Goldblum JR. Fluorescence in situ hybridization in the diagnosis of soft tissue neoplasms: a review. Adv Anat Pathol. 2009;16(6):383-391. PMID: 19851129, doi: 10.1097/PAP.0b013e3181bb6b86.

[11] Miettinen M. From morphological to molecular diagnosis of soft tissue tumors. Adv Exp Med Biol. 2006;587:99-113. PMID: 17163160. DOI: 10.1007/978-1-4020-5133-3_10. http://dx.doi.org/10.1007/978-1-4020-5133-3_10

[12] Mehra S, de la Roza G, Tull J, et al. Detection of the FOXO1 (FKHR) gene break-apart fluorescence in situ hybridization in formalin-fixed, paraffin-embedded alveolar rhabdomyosarcomas and its clinicopathologic correlation. Diagn Mol Pathol. 2008;17(1):14-20. PMID: 18303411. doi: 10.1097/PDM.0b013e3181255e62.

[13] Antonescu CR. Molecular profiling in the diagnosis and treatment of high grade sarcomas. Ultrastruct Pathol. 2008;32(2):37-42. PMID: 18446666. doi: 10.1080/01913120801897174. 